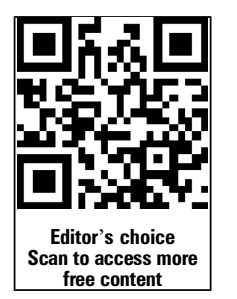

Correspondence to Dr Glenda Sobey, Head of EDS National Diagnostic Service, Sheffield Children's NHS Foundation Trust, Sheffield, UK; eds@sch.nhs.uk

Received 28 April 2014 Revised 4 June 2014 Accepted 9 June 2014 Published Online First 3 July 2014

\title{
Ehlers-Danlos syndrome: how to diagnose and when to perform genetic tests
}

\author{
Glenda Sobey
}

\section{ABSTRACT}

The term Ehlers-Danlos syndrome (EDS) encompasses a group of inherited connective tissue disorders. The manifestations of EDS can be seen in skin, joints, blood vessels and internal organs and vary from mild to severe and life threatening. Each subtype is a separate and different condition. The genetic basis of many subtypes has now been elucidated, confirming heterogeneity. An awareness of the different conditions within this group is the starting point towards accurate diagnosis. Accurate elicitation of history and clinical signs is vital in selecting the correct confirmatory investigation. Skin biopsy with electron microscopy can be helpful in the decision process of whether and when to perform genetic testing. Correct diagnosis within the EDSs allows targeted management, family screening and prenatal diagnosis.

\section{INTRODUCTION}

Ehlers-Danlos syndrome (EDS) is composed of separate and distinct entities. The underlying genetic defect, where known, is different in each subtype. Many of the clinical features are commonly seen between subtypes where skin, joints, blood vessels and internal organs are variably affected. Accurate clinical diagnosis with appropriate choice of genetic test can thus be challenging.

The correct diagnosis of EDS is further vexed by an outdated classification, confounding symptomatology and inaccuracy in eliciting clinical signs as these can be subtle. Furthermore, several types are rare and will be seen seldom or never in a physician's practice. Many types of EDS present in childhood. The presentation of vascular EDS as sudden death in adulthood is, however, well described as is the detection of affected but asymptomatic individuals picked up on cascade screening. Throughout life, there are some presenting features that should alert the physician to a possible EDS diagnosis (box 1).

\section{Box 1 When to consider the diagnosis of EDS}

Ehlers-Danlos syndrome needs to be considered when, in the absence of another explanation, one or more of the following occur:

- late walking with joint hypermobility

- abnormal bruising and bleeding

- unexplained vessel rupture or dissection

- tissue fragility, atrophic scarring or skin hyperextensibility

- symptomatic joint hypermobility \pm dislocations

- Hollow organ rupture
A systematic approach to the patient with suspected EDS is essential to avoid costly and unnecessary investigations, but first one needs to be aware of the different subtypes within this group and their diagnostic features.

\section{CLASSIFICATION}

Table 1 shows the Villefranche Classification of EDS together with subsequently identified subtypes and the molecular classification.

Identification of genetic changes affecting the synthesis and structure of type I, III and V collagen prompted the Villefranche Classification. ${ }^{1}$ This classification is still widely used. The previous classification of EDS using numbers, for example, EDS IV for vascular EDS, EDS I for classical EDS has been discarded as this was purely on clinical grounds.

The molecular genetic elucidation of further subtypes means that a new classification is required. ${ }^{2}$

Among the subtypes mentioned in table 1 , the classical, vascular and hypermobile types are most commonly seen. The other types mentioned are extremely rare.

\section{HISTORY}

The personal and family history are potentially contributory, and it is useful for a detailed pedigree to be completed. It is important not to miss other diagnoses that are sometimes confused with EDS and have their own specific treatment (box 2).

\section{EXAMINATION}

Clinical examination should include examination of the whole skin and assessment of the joints for hypermobility. The Beighton score (table 2) is the standard for recording this, and a score of 5 or more indicates a generalised joint hypermobility. ${ }^{3}$

\section{SPECIAL INVESTIGATIONS}

Skin biopsy is helpful in patients where particular subtypes are considered.

Skin biopsies can be taken for histology, electron microscopy and fibroblast culture. For patients who have had vascular events, do be aware that they may be on anticoagulants and respond according to surgical guidelines.

\section{CLASSICAL EDS}

Marked skin hyperextensibility (figure 1), together with widened atrophic cutaneous scars (figure 2) and generalised joint hypermobility, is typical of classical EDS (MIM 130010). ${ }^{4}$ Additional skin signs are easy bruising with staining due to haemosiderin deposition (particularly noticeable on the shins and dorsa of the hands), subcutaneous spheroids (fat lobules that have calcified after losing their blood 
Table 1 Ehlers-Danlos syndrome classification

\begin{tabular}{lll}
\hline Villefranche classification & Inheritance pattern \\
\hline Classical & Autosomal dominant \\
Hypermobile & Autosomal dominant \\
Vascular & Autosomal dominant \\
Kyphoscoliotic & Autosomal recessive \\
Arthrochalasic & Autosomal dominant & Autosomal recessive \\
Dermatosparactic & Inheritance pattern \\
\hline Subtype identified after Villefranche & Autosomal recessive \\
\hline Tenascin-X deficient & Autosomal recessive \\
Ehlers-Danlos syndrome with scoliosis, myopathy, hearing impairment & Autosomal recessive \\
Musculocontractural Ehlers-Danlos syndrome & Targeted COL1A1,COL1A2 Procollagen N-proteinase (ADAMTS2) \\
\hline
\end{tabular}

supply) and molluscoid pseudotumours (associated with scars over the knees and elbows that are thickened fleshy skin lesions (figure 3)). Stretch marks (striae) are absent in classical EDS.

Where classical EDS is suspected, a skin biopsy for electron microscopy is the recommended first step in investigation.

The typical electron microscopy findings in classical EDS are termed 'collagen flowers' or 'cauliflowers'. These result from abnormal fibrillogenesis (figure 4). When multiple collagen flowers are seen, a diagnosis of classical EDS is suspected and the next step is molecular genetic testing of type $\mathrm{V}$ collagen. The COL5A1 and COL5A2 genes are implicated. Mutations in type $\mathrm{V}$ collagen have long been known to cause classical EDS. ${ }^{5}$ Reduced availability of type $\mathrm{V}$ collagen is thought to be the major disease causing mechanism. ${ }^{6}$ A large number of different mutations have been identified in type $\mathrm{V}$ collagen in patients with classical EDS. As yet, there is no obvious genotype-phenotype correlation.

Classical EDS is a dominantly inherited condition. Severity is seen to vary significantly, even within the same family.

\section{Recommendations for classical EDS management}

- Cardiology assessment including echocardiogram.

- Regular cardiology follow-up.

- Joint hypermobility managed by rheumatology including physiotherapy and occupational therapy.

- Shin pads helpful to reduce injury in childhood.

- Patient should be known to local plastic surgeons for expert suturing of any wounds.

- Wear medical warning bracelet inscribed 'classical EDS'.

- Patient to carry information leaflet on the condition to provide to school, employer, healthcare staff and other appropriate contacts.

\section{Box 2 Conditions mistaken for Ehlers-Danlos syndrome}

- Osteogenesis imperfecta

- Loeys-Dietz syndrome

- Skeletal dysplasias

- Mucopolysaccharidoses

- Cutis laxa

- Pseudoxanthoma elasticum

- Ullrich congenital muscular dystrophy

- Bethlem myopathy

\section{VASCULAR EDS}

The diagnosis of vascular EDS (MIM 130050) carries with it the life-threatening risks of blood vessel and organ rupture, sometimes in childhood. The clinical features typical of vascular EDS may be subtle or absent, making diagnosis difficult particularly where there is no positive family history. Sudden death in the third or fourth decade of life can be the presenting feature.

\section{Features of vascular EDS}

Easy bruising: This can be seen at sites not prone to trauma and is often the presenting sign in childhood. Child protection agencies may have been involved due to this prominent feature.

Skin: This is typically thin and translucent with visible underlying vessels. The skin is not hyperextensible but can be fragile (figure 5).

Facial features: In some patients, the typical features of a thin pinched nose, prominent eyes and lobeless ears together with a lack of subcutaneous fat are so striking that the diagnosis may be suspected on this aspect alone.

Acrogeria: A prematurely aged appearance particularly to the hands and feet due to thin wrinkled skin is sometimes seen.

Joint hypermobility: In vascular EDS, this is usually limited to the small joints of the hands.

Congenital talipes: May be associated.

Repeated pneumothoraces ${ }^{7}$ : Can be the initial presentation.

A diagnosis of vascular EDS needs to be excluded in any patient where an unexplained arterial rupture or bowel rupture has occurred. ${ }^{8}$ This is equally important where the diagnosis is not vascular EDS as a wide range of interventional procedures (which would be contraindicated and potentially lethal in vascular EDS) is then available to the patient.

The major risks for patients with vascular EDS are arterial (including aortic) dissection, rupture and aneurysm. The sigmoid colon is the commonest site for bowel rupture-this

Table 2 The Beighton score for joint hypermobility

\begin{tabular}{|c|c|c|}
\hline Joint mobility & Unilateral & Bilatera \\
\hline Passive dorsiflexion of fifth finger beyond $90^{\circ}$ & 1 & 2 \\
\hline Passive flexion of thumb to forearm & 1 & 2 \\
\hline Hyperextension of the elbow beyond $10^{\circ}$ & 1 & 2 \\
\hline Hyperextension of the knee beyond $10^{\circ}$ & 1 & 2 \\
\hline $\begin{array}{l}\text { Forward flexion of the trunk with knees fully extended } \\
\text { and palms resting on the floor }\end{array}$ & Present $=1$ & \\
\hline
\end{tabular}




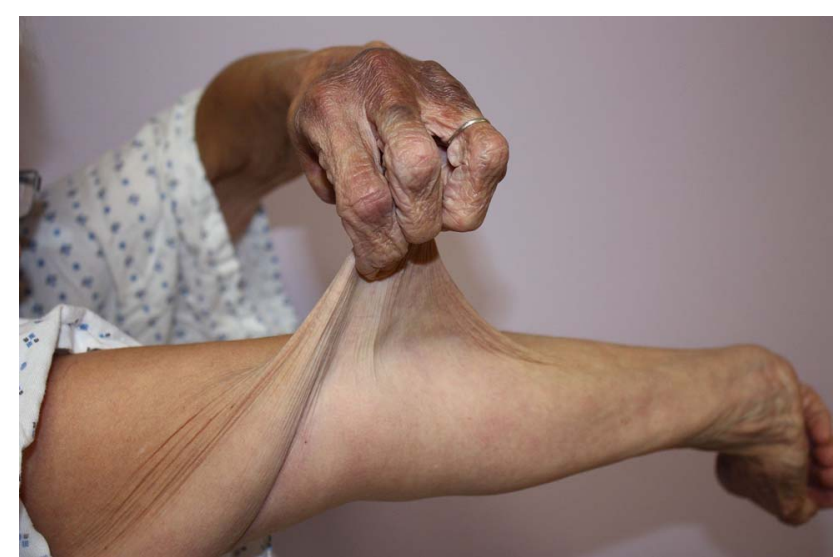

Figure 1 Persistent haemosiderin staining on hand and skin hyperextensibility at forearm.

can be seen at all ages. Obstetric complications include uterine and arterial rupture as well as massive postpartum haemorrhage and severe lacerations from tearing at vaginal delivery.

Vascular EDS is caused by mutations in the COL3A1 gene that encodes type III collagen. Molecular genetic testing is highly sensitive and specific for vascular EDS. Accurate prognostication by genotype-phenotype correlation has been extensively investigated but is not yet reliable. Parental mosaicism is recognised and can explain unexpected inheritance patterns. ${ }^{9}$ There are also characteristic changes on electron microscopy of skin with marked collagen diameter variability (figure 6).

\section{Recommendations for vascular EDS medical management}

- Surgical and endovascular interventions are discouraged and conservative medical management preferred where possible.

- Wear medical warning bracelet inscribed 'vascular EDS' with information on specific emergency care given to the organisation supplying the bracelet.

- Regular follow-up in cardiology is advised, preferably in a specialised unit. The role of imaging and medication is still in the early stages of use and evidence is being gathered. Medication aimed at reducing the incidence of arterial rupture or dissection has been trialled. ${ }^{10}$

- Need for psychological support following the diagnosis with special attention to the needs of children and adolescents requiring transitional care.

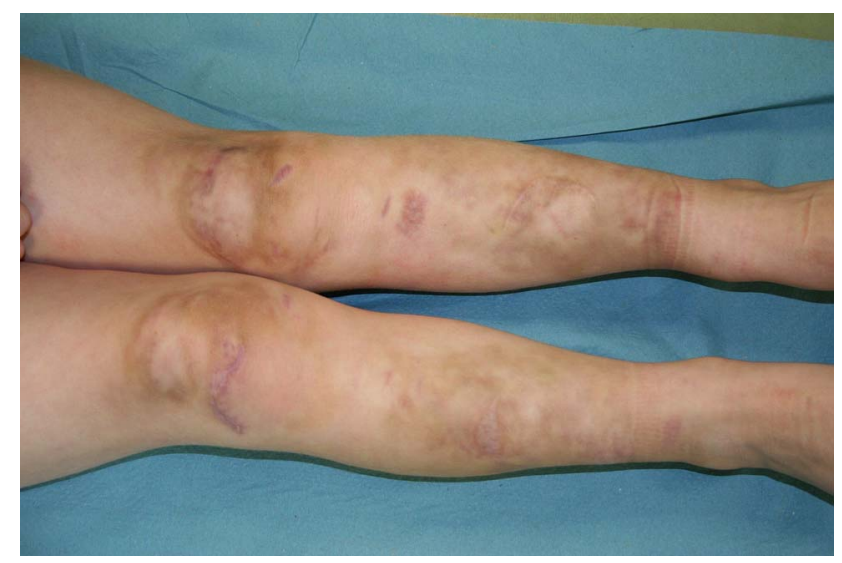

Figure 2 Bruising and atrophic scars of the knees and shins.

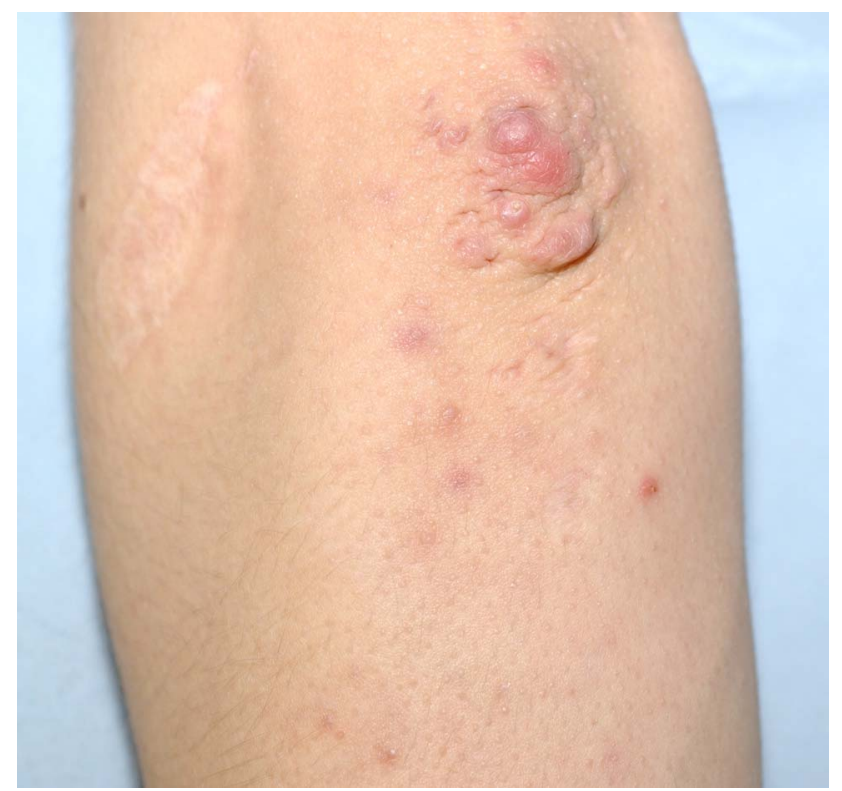

Figure 3 Molluscoid pseudotumour of elbow.

Recommendations for vascular EDS self-management

- Avoid potentially harmful activities, for example, contact sports, heavy lifting, rapid acceleration and deceleration.

- Make school, family, employers aware of the condition and appropriate emergency management.

- Follow healthy lifestyle choices.

\section{HYPERMOBILE EDS}

There is no diagnostic test for this condition, and the genetic basis is still unknown. Add to this the fact that joint hypermobility is reasonably common (thought to be up to $10 \%$ in the general population) and that even within the same family the manifestations can vary and it is clear why accurate delineation remains elusive. There is still much debate whether hypermobile EDS (MIM 130020) and joint hypermobility syndrome represent the same condition. Joint hypermobility does appear to be dominantly inherited in many families.

The Beighton score is useful in confirming the presence and extent of joint hypermobility, although this score can often decrease with age, injury, surgery and the effects of arthritis.

Although patients in this group are not prone to lifethreatening complications, the effects can be debilitating in

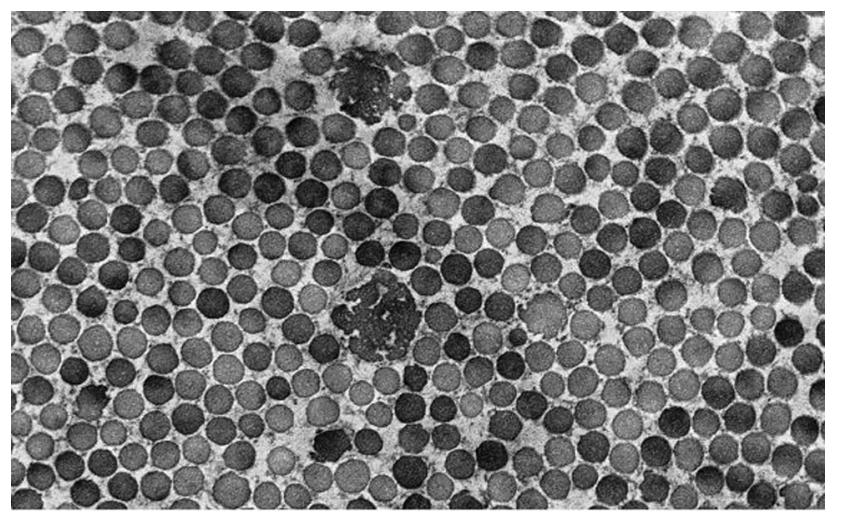

Figure 4 Classical Ehlers-Danlos syndrome electron microscopy of the skin showing the characteristic collagen flowers. 


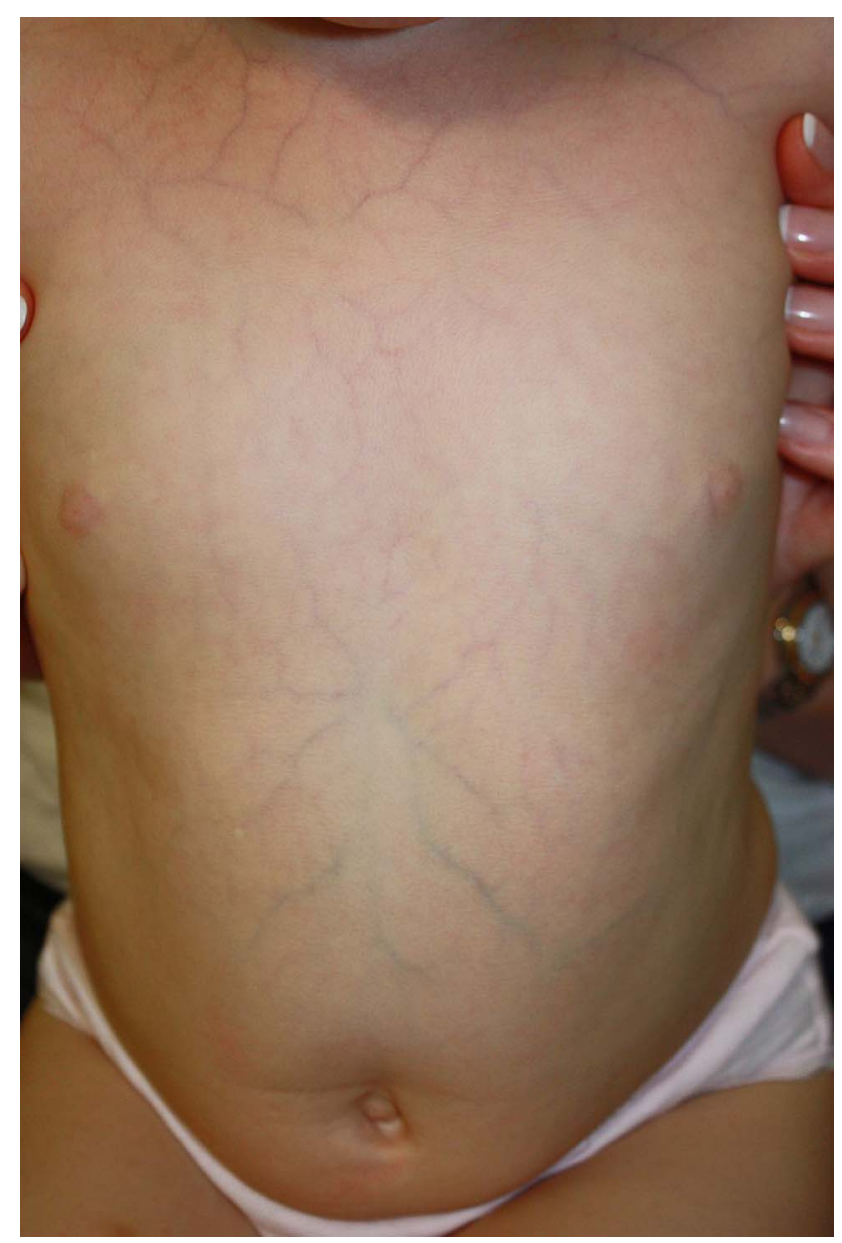

Figure 5 Translucent skin with visible veins in vascular Ehlers-Danlos syndrome.

the extreme. The combination of severe chronic joint pain and the symptoms of autonomic dysfunction can severely impair quality of life. ${ }^{11}$ Gastrointestinal and urinary symptoms are also commonly reported. Women present more commonly with this condition than men-this is still unexplained. ${ }^{12}$

Postural orthostatic tachycardia syndrome (POTS) is well recognised in this group. POTS is a syndrome of dysautonomia and has a wide range of symptoms that occur particularly on

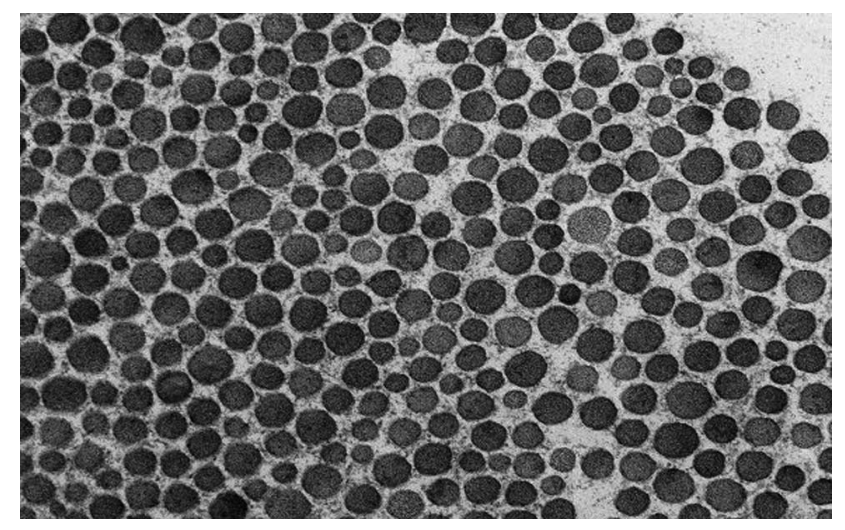

Figure 6 Vascular Ehlers-Danlos syndrome electron microscopy of the skin; marked variability of the dimensions of the collagen bundles is evident. postural change or standing. ${ }^{13}$ Transient loss of consciousness, dizziness, palpitations, chest pain and shortness of breath are all recognised.

\section{Recommendations for hypermobile EDS management}

- Referral to rheumatology for assessment of appropriate rehabilitation by physiotherapy and occupational therapy.

- Following advice of physiotherapy, regular graded exercise is helpful.

- Consider a referral to a multidisciplinary pain clinic where chronic pain is a significant component of morbidity.

- Actively reassure patient that they do not have a worrying type of EDS.

- Put patient in touch with appropriate support group.

\section{TENASCIN X-DEFICIENT EDS}

The autosomal-recessive condition of Tenascin X-deficient EDS has features of marked skin laxity, pronounced joint hypermobility and severe bruising. ${ }^{14}$ The abnormal scarring seen in classical EDS is absent. Electron microscopy is sometimes helpful.

The parents (particularly mothers) of affected individuals, who have a single Tenascin $\mathrm{X}$ mutation themselves, can have features of joint hypermobility. ${ }^{15}$ There is no evidence to suggest that this is a common cause for hypermobile EDS.

\section{KYPHOSCOLIOTIC EDS}

This autosomal-recessive condition (MIM 225400) is characterised by early-onset, progressive kyphoscoliosis, hypotonia and gross motor delay as well as joint hypermobility, skin hyperextensibility, easy bruising, atrophic scarring and fragility of the sclerae with raised intraocular pressure. Vascular rupture is reported. ${ }^{16} 17$ Cognitive development is normal. Because of severe muscle hypotonia and delayed motor development, children with this condition are often thought to have a primary neuromuscular disease.

It is caused by deficiency in the enzyme lysyl hydroxylase. ${ }^{18}$ The diagnosis can be confirmed by showing an increased ratio of lysylpyridinoline to hydroxylysylpyridinoline crosslinks in the urine. These test results correlate fully with the presence of a homozygous or compound heterozygous mutation in the PLOD1 gene.

\section{FKBP14 EDS}

This autosomal-recessive condition (MIM 614557) should be considered in all patients thought to have kyphoscoliotic EDS, but where urine crosslinking is normal and no mutations are found in PLOD1, particularly if there is sensorineural hearing impairment. ${ }^{19}$ The severe generalised hypotonia at birth with marked muscle weakness usually improves in infancy.

\section{CHST14 EDS/MUSCULOCONTRACTURAL EDS}

The features of this rare condition (MIM 601776) include progressive kyphoscoliosis, adducted thumbs in infancy, clubfoot, arachnodactyly, joint hypermobility, fragile and hyperextensible skin with atrophic scars and delayed wound healing. ${ }^{20-22}$

\section{ARTHROCHALASIC EDS}

Arthrochalasic EDS (MIM 130060) is extremely rare. ${ }^{23}$ Congenital bilateral hip dislocation, severe joint hypermobility with recurrent dislocations, skin hyperextensibility, easy bruising, atrophic scars, kyphoscoliosis and osteopaenia are the features of this autosomal-dominant condition. It is caused by mutations in type I collagen that lead to loss of exon 6 or part thereof. 


\section{DERMATOSPARACTIC EDS}

Dermatosparactic EDS (MIM 225410) is extremely rare. This autosomal-recessive condition is caused by deficient activity of the procollagen-N-proteinase encoded by the ADAMTS2 gene. The clinical features include redundant, sagging, fragile skin, delayed closure of the fontanelles, blue sclerae, umbilical hernia and short stature. ${ }^{24}$ Most patients have a very severe phenotype presenting at birth, but some have milder manifestations. ${ }^{25}$ Electron microscopy of skin shows characteristic 'hieroglyphic fibrils'. ${ }^{26}$ The diagnosis is confirmed through genetic testing of ADAMTS2.

\section{CONCLUSION}

There are now a wide range of EDS subtypes recognised clinically, which can be confirmed by molecular genetic testing. We appreciate now that these are multisystem disorders. Clinical features are shared between some subtypes making diagnosis of these rare conditions challenging. As these conditions are individually rare, the decision when to perform genetic tests can be difficult and when the clinical diagnosis is in doubt referral to specialist centres is the most efficient way forward.

The development of next-generation sequencing platforms will hasten the molecular genetic testing process and make it more economical, but the clinician needs to be aware of EDS and its variable presentations to consider it as a potential cause. The firm diagnosis of EDS, in some forms life threatening, enables genetic counselling and targeted management to optimise both quality of life and life expectancy in this group of rare disorders.

\section{Competing interests None.}

Patient consent Obtained.

Provenance and peer review Commissioned; externally peer reviewed.

\section{REFERENCES}

1 Beighton P, De Paepe A, Steinmann B, et al. Ehlers-Danlos Syndrome: revised nosology, Villefranche, 1997. Am J Med Genet 1998;77:31-7.

2 De Paepe A, Malfait F. The Ehlers-Danlos Syndrome, a disorder with many faces. Clin Genet 2012;82:1-11.

3 Beighton P, Horan F. Orthopaedic aspects of the Ehlers Danlos Syndrome. J Bone Jt Surg 1969:51:444-53.

4 Ritelli M, Dordoni C, Venturini M, et al. Clinical and molecular characterization of 40 patients with classic Ehlers-Danlos syndrome: identification of 18 COL5A1 and 2 COL5A2 novel mutations. J Rare Dis 2013;8:58.

5 De Paepe A, Nuytinck L, Hausser I, et al. Mutations in the COL5A1 gene are causal in the Ehlers-Danlos Syndrome I and II. Am J Hum Genet 1997;60:547-54.

6 Symoens S, Syx D, Malfait F, et al. Comprehensive molecular analysis demonstrates type $V$ collagen mutations in over $90 \%$ of patients with classic EDS and allows to refine diagnostic criteria. Hum Mutat 2012;33:1485-93.
7 Foulkes AC, Pollitt R, Sobey $\mathrm{G}$, et al. Palmoplantar contractures in childhood: a rare complication of vascular Ehlers-Danlos syndrome. Clin ExpDermatol 2013;38:517-19.

8 Singh M, Puppala S, Pollitt RC, et al. Femoral artery dissection in vascular type Ehlers-Danlos syndrome; leave well alone? Eur J Vasc EndovascSurg 2012:43:341-2.

9 Palmeri S, Mari F, Meloni I, et al. Neurological presentation of Ehlers Danlos Syndrome type IV in a family with parental mosaicism. Clin Genet 2003;63:510-15.

10 Ong KT, Perdu J, De Backer J, et al. Effect of celiprolol on prevention of cardiovascular events in Ehlers-Danlos Syndrome: a prospective, randomised, open, blind-endpoints trial. Lancet 2010;376:1476-84.

11 Farmer AD, Fikree A, Aziz Q. Addressing the confounding role of joint hypermobility syndrome and gastrointestinal involvement in postural orthostatic tachycardia syndrome. Clin Auton Res 2014;24:157-8.

12 Rombaut L, Malfait F, Cools A, et al. Musculoskeletal complaints, physical activity and health- related quality of life among patients with the Ehlers Danlos Syndrome hypermobility type. Disabil Rehabil 2010;32:1339-45.

13 Benarroch EE. Postural tachycardia syndrome: a heterogeneous and multifactorial disorder. Mayo Clin Proc 2012;87:1214-25. http://dx.doi.org/10.1016/j.mayocp. 2012.08.013 (accessed 1 Apr 2014).

14 Schalkwijk J, Zweers MC, Steijlen PM, et al. A recessive form of the Ehlers-Danlos syndrome caused by tenascin-X deficiency. N Engl J Med 2001;345:1167.

15 Zweers MC, Bristow J, Steijlen PM, et al. Haploinsufficiency of TNXB is associated with hypermobility type of Ehlers-Danlos Syndrome. Am J Hum Genet 2003;73:214-17.

16 Giunta C, Randolph A, Steinmann B. Mutation analysis of the PLOD1 gene: an efficient multistep approach to the molecular diagnosis of the kyphoscoliotic type of Ehlers-Danlos syndrome (EDS VIA). Mol Genet Metab 2005;86:269-76.

17 Giunta C, Randolph A, Al-Gazali LI, et al. Nevo syndrome is allelic to the kyphoscoliotic type of the Ehlers-Danlos syndrome (EDS VIA). Am J Med Genet 2005;133A:158-64.

18 Pinnell SR, Krane SM, Kenzora JE, et al. A heritable disorder of connective tissue. Hydroxylysine-deficient collagen disease. N Engl J Med 1972;286:1013-20.

19 Baumann M, Giunta C, Krabichler B, et al. Mutations in FKBP14 Cause a Variant of Ehlers Danlos Syndrome with progressive kyphoscoliosis, myopathy and hearing loss. Am J Hum Genet 2012;90:201-16.

20 Dündar M, Müller T, Zhang Q, et al. Loss of dermatan-4-sulfotransferase 1 function results in adducted thumb-clubfoot syndrome. Am J Hum Genet 2009;85:873-82.

21 Miyake N, Kosho T, Mizumoto S, et al. Loss-of-function mutations of CHST14 in a new type of Ehlers-Danlos syndrome. Hum Mutat 2010;31:966-74.

22 Kosho T, Miyake N, Hatamochi A, et al. A new Ehlers-Danlos syndrome with craniofacial characteristics, multiple congenital contractures, progressive joint and skin laxity, and multisystem fragility-related manifestations. Am J Med Genet 2010;152A:1333-46.

23 Steinmann B, Royce PM, Superti-Furga A. The Ehlers-Danlos Syndrome from connective tissue and its heritable disorders. Royce PM, Steinmann B: Wiley-Liss, New York: 2002:431-523.

24 Malfait F, De Coster P, Hausser I, et al. The natural history, including orofacial features of three patients with Ehlers-Danlos syndrome, dermatosparaxis type (EDS type VIIC). Am J Med Genet 2004;131:18-28.

25 Solomons J, Coucke P, Symoens S, et al. Dermatosparaxis (Ehlers-Danlos type VIIC): prenatal diagnosis following a previous pregnancy with unexpected skull fractures at delivery. Am J Med Genet 2013;161A:1122-5.

26 Piérard GE, Lapière M. Skin in dermatosparaxis. Dermal microarchitecture and biomechanical properties. J Invest Dermatol 1976;66:2-7. 